\title{
NTPDase1/CD39 and aberrant purinergic signalling in the pathogenesis of COPD
}

\author{
Zsofia Lazar 1,2,7, Nina Müllner ${ }^{1,7}$, Monica Lucattelli3,7, Cemil Korcan Ayata ${ }^{1,7}$, \\ Sanja Cicko ${ }^{1}$, Gennady G. Yegutkin ${ }^{4}$, Giovanna De Cunto ${ }^{3}$, Tobias Müller ${ }^{1}$, \\ Anja Meyer ${ }^{1}$, Madelon Hossfeld ${ }^{1}$, Stephan Sorichter ${ }^{1}$, Ildiko Horvath ${ }^{2}$, \\ Christian J. Virchow ${ }^{5}$, Simon C. Robson ${ }^{6}$, Giuseppe Lungarella ${ }^{3}$ and Marco Idzko ${ }^{1}$
}

Affiliations: ${ }^{1}$ Dept of Pulmonology, University Hospital Freiburg, Freiburg, Germany. ${ }^{2}$ Dept of Pulmonology, Semmelweis University, Budapest, Hungary. ${ }^{3}$ Dept of Life Sciences, University of Siena, Siena, Italy. ${ }^{4}$ MediCity Research Laboratory, University of Turku, Turku, Finland. ${ }^{5}$ Dept of Pulmonology, University Hospital Rostock, Rostock, Germany. ${ }^{6}$ Division of Gastroenterology, Dept of Medicine, Beth Israel Deaconess Medical Center, Harvard Medical School, Boston, MA, USA. ${ }^{7}$ These authors contributed equally.

Correspondence: Marco Idzko, Dept of Pulmonary Medicine, University Hospital Freiburg, Killianstraße 5, 79106-Freiburg, Germany.

E-mail: marco.idzkoduniklinik-freiburg.de

ABSTRACT Purinergic receptor activation via extracellular ATP is involved in the pathogenesis of chronic obstructive pulmonary disease (COPD). Nucleoside triphosphate diphosphohydrolase-1/CD39 hydrolyses extracellular ATP and modulates P2 receptor signalling.

We aimed to investigate the expression and function of CD39 in the pathogenesis of cigarette smokeinduced lung inflammation in patients and preclinical mouse models. CD39 expression and soluble ATPase activity were quantified in sputum and bronchoalveolar lavage fluid (BALF) cells in nonsmokers, smokers and COPD patients or mice with cigarette smoke-induced lung inflammation. In mice, pulmonary ATP and cytokine concentrations, inflammation and emphysema were analysed in the presence or absence of CD39.

Following acute cigarette smoke exposure CD39 was upregulated in BALF cells in smokers with further increases in COPD patients. Acute cigarette smoke exposure induced CD39 upregulation in murine lungs and BALF cells, and ATP degradation was accelerated in airway fluids. CD39 inhibition and deficiency led to augmented lung inflammation; treatment with ATPase during cigarette smoke exposure prevented emphysema.

Pulmonary CD39 expression and activity are increased in COPD. CD39 deficiency leads to enhanced emphysema in mice, while external administration of a functional CD39 analogue partially rescues the phenotype. The compensatory upregulation of pulmonary CD39 might serve as a protective mechanism in cigarette smoke-induced lung damage.

@ERSpublications

The upregulation of pulmonary ATPase is a protective mechanism in cigarette smoke-induced lung inflammation http://ow.ly/S5YcC

\section{This article has supplementary material available from erj.ersjournals.com}

Received: Nov 212014 | Accepted after revision: Aug 052015 | First published online: Nov 052015

Support statement: M. Idzko is a recipient of grants from the German Research Foundation (Deutsche Forschungsgemeinschaft (DFG), grant ID 7/4-1) and the Boehringer Ingelheim Foundation. Z. Lazar was a recipient of the European Respiratory Society long-term research fellowship (grant ID 50-2012). Funding information for this article has been deposited with FundRef.

Conflict of interest: None declared.

Copyright OERS 2016 


\section{Introduction}

Chronic obstructive pulmonary disease (COPD) is a leading cause of morbidity and mortality worldwide [1]. It is associated with the specific inflammation of small airways, which results in airway obstruction, parenchymal destruction and the development of emphysema. Although inhalation of cigarette smoke is the main risk factor for the development of COPD, the precise mechanisms initiating and perpetuating the disease and the underlying inflammation are poorly understood.

An increasing body of evidence supports the involvement of endogenous nucleotides, which activate P2 receptor subtypes, in neutrophilic airway inflammation [2-5]. More specifically, increased pulmonary levels of ATP can be found in mice and human subjects following exposure to cigarette smoke, and also in patients with COPD. Importantly, ATP concentration in the bronchoalveolar lavage fluid (BALF) of patients correlates with neutrophil counts and airflow limitation [3]. In the mouse model, inhibition of specific P2 receptor subtypes has been shown to decrease cigarette smoke-induced lung inflammation and emphysema [6, 7].

The levels of nucleotides in mammalian tissues are tightly controlled by the nucleoside triphosphate diphosphohydrolases (NTPDases) 1-8 and other ectonucleotidases. CD39/NTPDase1/ecto-apyrase is the dominant purinergic ecto-enzyme in the immune and vascular barrier in the lung, and it is also a key regulator of the functionality of immune cells [8]. It regulates ATP-mediated P2 receptor signalling by hydrolysing ATP/ $\mathrm{ADP}$ to AMP, which is then further metabolised by CD73/ecto-5'-nucleotidase to adenosine [9].

Interestingly, besides its general ecto-enzymatic function, CD39 might directly modulate the function of immune cells such as neutrophils, macrophages or dendritic cells $[10,11]$. Consequently, an important role of CD39 in inflammatory disorders, including acute respiratory distress syndrome, hyperoxic acute lung injury, colitis and autoimmune diabetes has been demonstrated [12, 13]. Recently, we have shown that CD39 is involved in the pathogenesis of allergic airway inflammation by modulating dendritic cell function [14]. However, the role and regulation of CD39 in the development and maintenance of cigarette smoke-induced lung inflammation and emphysema is unknown.

The aim of this study was to investigate, for the first time, the expression and functional impact of CD39 on the pathogenesis of COPD. For this purpose we measured the expression of CD39 and ATPase in sputum and/or BALF of nonsmokers, smokers, ex-smokers and patients with COPD, as well as in BALF and lung tissue of mice with cigarette smoke-induced lung inflammation. In order to elucidate the functional relevance of these findings, the effect of CD39 inhibition/deficiency on the development of cigarette smoke-induced lung inflammation and lung emphysema was analysed in a translational mouse model. Finally, to support the potential therapeutic implications of these findings, cigarette smoke-exposed mice were treated with a functional analogue of CD39 and the degree of lung inflammation and lung emphysema was determined.

\section{Methods}

\section{Collection of BALF and sputum from control subjects and patients with COPD}

BALF was collected from control subjects and ex-smoking COPD patients as part of a previous study and processed as described previously [3]. A subset of smokers underwent bronchoscopy and bronchoalveolar lavage after a 4-h period of smoking deprivation (no smoke exposure) and immediately after a 4-h period during which eight cigarettes were smoked (acute smoke exposure), with a 2-week interval in between. Induced or spontaneous sputum was collected from control subjects, as previously reported [15]. Patients were ex-smokers (abstinence $>6$ months prior to recruitment) and had known COPD which had been established previously by a respiratory specialist and were in stable condition. Spirometric classification was performed according to RABE et al. [1]. Detailed information on eligibility criteria, patient characteristics and the procedure is given in the online supplementary material.

The study was conducted in line with the recommendations of the Declaration of Helsinki and was approved by the local ethics committee of University Medical Centre (Freiburg, Germany). All participants gave written informed consent.

Mice

C57BL/6 mice (aged 6-8 weeks) were purchased from Charles River (Calco, Italy) or were bred at the animal facilities at the University Hospital Freiburg. $C d 39$-deficient mice $\left(C d 39^{-/-}\right)$on a $\mathrm{C} 57 \mathrm{BL} / 6$ background were generated as previously described [14]. All experiments were performed according to institutional guidelines of the animal ethics committees of the Italian or German governments.

\section{Animal models of acute and chronic cigarette smoke-induced lung inflammation}

The induction of acute and chronic smoke-associated lung inflammation was performed as previously described $[6,7,16]$. In some experiments, intratracheal or intraperitoneal treatment was performed prior to smoke exposure. BALF and lungs were collected and processed as previously described [3, 7]. Additional details are provided in the online supplementary material. 


\section{Enzyme histochemistry on murine lungs}

For localisation of ATPase activity, the lead phosphate method was applied [17], which is based on the precipitation of lead by free phosphate generated from ATPase activity. The intensity of lead staining was quantified using ImageJ v1.46r software (National Institutes of Health, Bethesda, MD, USA). Further details are available in the online supplementary material.

\section{ATP and cytokine measurements in BALF and sputum supernatant}

Cell-free human sputum and murine BALF supernatants were incubated for $30 \mathrm{~min}$ at room temperature with $100 \mathrm{mM}$ and $100 \mathrm{nM}$ ATP, respectively. ATPase activity is shown as percentage of ATP hydrolysed (100 - percentage ATP recovered). ATP concentration was determined as previously described $[3,18]$. Cytokine concentrations in BALF were measured using commercially available ELISA kits (R\&D Systems, Minneapolis, MN, USA).

\section{Real-time PCR}

Total RNA was isolated using QIAzol (Qiagen, Hilden, Germany); complementary DNA synthesis was performed using a First Strand cDNA synthesis kit (ThermoScientific, Bremen, Germany). Quantitative PCR was performed using a LightCycler 480 (Roche, Mannheim, Germany) using a fast-blue+UNG kit (Eurogentec, Cologne, Germany). $\beta 2 M$ and GAPDH were used as reference genes. Gene expression assay design and analysis were performed as previously described [19]; primer/probe sequences are available upon request.

\section{Statistical analysis}

Sputum data were not normally distributed (D'Agostino-Pearson test) and were analysed using nonparametric tests (Kruskal-Wallis with Dunn's post hoc test, Spearman correlation) and reported as median (interquartile range). CD39 expression data in sputum were logarithmically transformed to yield normal distribution. Otherwise, a one-sample t-test, unpaired t-test or ANOVA with post hoc Bonferroni test were applied and data are shown as mean \pm SEM (GraphPad Prism 5; GraphPad Software, San Diego, CA, USA). Differences were considered significant if $\mathrm{p}<0.05$.

\section{Results}

CD39 expression of airway inflammatory cells is modulated by smoking and increased in COPD

To analyse the effects of smoking on CD39 expression and activity in the airways, BALF leukocytes collected in a previously published cohort of nonsmokers, smokers and patients with COPD were studied [3]; subject characteristics are shown in table 1 . We observed a tendency to increased expression of CD39 on BALF leukocytes from patients compared to smokers and nonsmokers ( $p=0.09$; fig. 1a). Previously, we reported that in human smokers BALF ATP levels were strongly increased after acute smoke exposures compared to a period of smoking cessation [3]. Thus we questioned whether CD39 expression in BALF cells might also be influenced. As shown in figure $1 \mathrm{~b}$, a 4-h period of smoking induced a strong upregulation of CD39 expression in BALF cells of human smokers $(\mathrm{n}=8)$.

To support these findings in a broader cohort, sputa were collected from control subjects and ex-smoking patients with COPD. Patient characteristics and sputum variables are presented in table 2. As shown in fig. 1c, CD39 expression of total sputum cells was increased in current smokers compared to non- and ex-smoking control subjects. In fact, CD39 expression was higher in ex-smoking patients with COPD $(\mathrm{n}=26)$ than in

TABLE 1 Subject characteristics in the bronchoalveolar lavage fluid (BALF) study

\begin{tabular}{lccc} 
& \multicolumn{2}{c}{ Control subjects } & COPD patients \\
\cline { 2 - 3 } & Nonsmoking & Smoking & \\
\hline Subjects & 7 & 10 & 8 \\
Male/female & $2 / 6$ & $2 / 8$ & $0 / 8$ \\
Age years & $42 \pm 7$ & $37 \pm 7$ & $55 \pm 5^{* *} \#$ \\
Pack-years & $\mathrm{NA}$ & $10 \pm 4$ & $35 \pm 8^{\#}$ \\
GOLD severity I/II/III & $\mathrm{NA}$ & $\mathrm{NA}$ & $2 / 4 / 2$ \\
Leukocytes $\mathbf{\times 1 0 ^ { 6 }}$ cells-100 $\mathbf{~ m L}^{-1}$ BALF & $9.4 \pm 6.6$ & $13.7 \pm 10.7$ & $31.9 \pm 16.3$ \\
FEV $\%$ predicted & $102 \pm 8$ & $104 \pm 10$ & $70 \pm 15$ \\
\hline
\end{tabular}

Data are presented as $\mathrm{n}$ or mean $\pm \mathrm{SD}$. ANOVA with Bonferroni post hoc test or t-test were used. COPD: chronic obstructive pulmonary disease; GOLD: Global Initiative for Chronic Obstructive Lung Disease; FEV1: forced expiratory volume in $1 \mathrm{~s}$; NA: not applicable. ${ }^{* *}$ : $p<0.01$ versus nonsmoking control; ${ }^{\#}: p<0.01$ versus currently smoking control. 

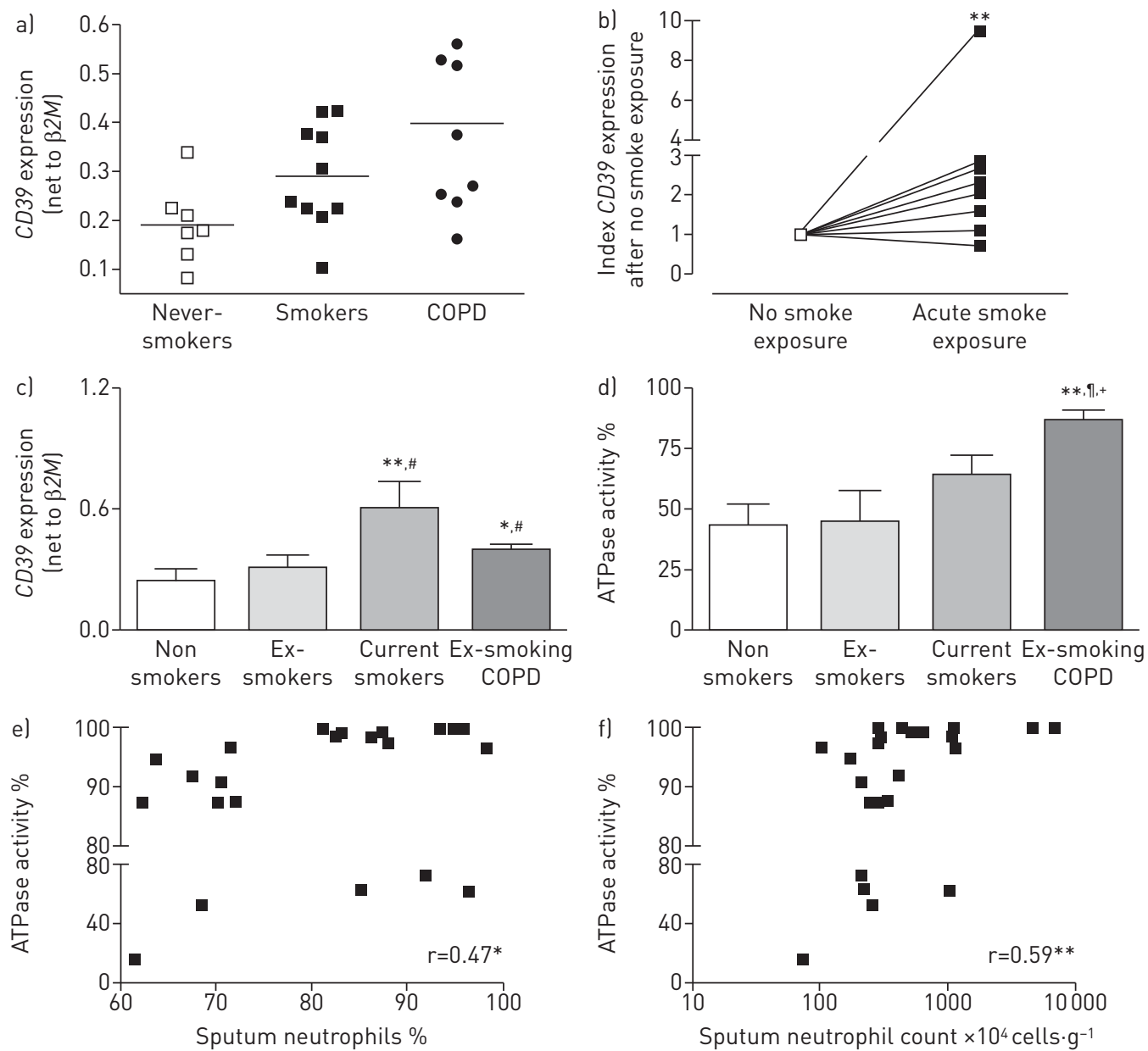

FIGURE 1 Cellular CD39 expression and soluble ATPase activity in airway inflammatory cells of nonsmokers, ex-smokers, current smokers and patients with chronic obstructive pulmonary disease (COPD). a) Expression of CD39 in total bronchoalveolar lavage fluid (BALF) cells isolated from never-smoking controls, smokers and patients with COPD was measured using quantitative (q)PCR; $p=0.09$. b) Effect of acute cigarette smoke inhalation on CD39 expression in BALF leukocytes of human smokers; ${ }^{* *}$ : $p<0.01$. c) Expression of CD39 in total sputum cells isolated from controls and ex-smoking patients with COPD was quantified using qPCR. Data are presented as mean \pm SEM. ${ }^{*}$ : $p<0.05 ;{ }^{* *}$ : $p<0.01$ nonsmoker versus current smoker, nonsmoker versus ex-smoking COPD; ${ }^{\#}$ : $p<0.05$ ex-smoker versus current smoker, ex-smoker versus ex-smoking COPD. d) Freshly prepared cell-free sputum supernatant was incubated with $100 \mathrm{mM}$ ATP for $30 \mathrm{~min}$ at room temperature, then ATPase activity was measured in a bioluminescent assay. Data are presented as mean \pm SEM. ${ }^{* *}$ : $p<0.01$ nonsmoker versus ex-smoking COPD; ๆ: $p<0.01$ ex-smoker versus ex-smoking COPD, ${ }^{+}: p<0.05$ current smoker versus ex-smoking COPD. e, f) Correlation of ATPase activity in sputum supernatant to e) percentages and f) counts of sputum neutrophils in ex-smoking patients with COPD. ${ }^{*}: p<0.05 ;{ }^{* *}: p<0.01$.

control subjects. Furthermore, the activity of ATPase in cell-free sputum supernatant was enhanced in patients $(\mathrm{n}=23)$ compared to nonsmokers, ex-smokers and current smokers (fig. 1d). Of note, ATPase activity was increased in patients with severe/very severe COPD compared to subjects with mild/moderate disease ( $\mathrm{p}=0.03$, data not shown), and showed a negative correlation with FEV1 \% predicted $(\mathrm{p}=0.01, \mathrm{r}=-0.52)$, which suggests that more severe airflow limitation is associated with the accelerated activity of airway ATPase. However, no relationship was observed between CD39 expression and disease severity or FEV1 (data not presented). Interestingly, in patients, ATPase activity showed a significant positive correlation to leukocyte count. $\mathrm{g}^{-1}$ sputum $(\mathrm{p}<0.01, \mathrm{r}=0.57)$, sputum neutrophil percentages (fig. 1e) and neutrophil counts (fig. lf), but not to the counts or percentages of macrophages, eosinophils and lymphocytes (data not shown).

\section{CD39 expression and pulmonary ATPase activity are enhanced in a mouse model of cigarette smoke-induced lung inflammation}

To corroborate the human findings, Cd39 expression and ATP hydrolysing activity were studied in BALF cells and supernatants in a mouse model for cigarette smoke-induced lung inflammation. Similarly to the human data, we observed an increase in Cd39 expression and soluble ATPase activity in BALF cells and 
TABLE 2 Subject characteristics in the sputum study

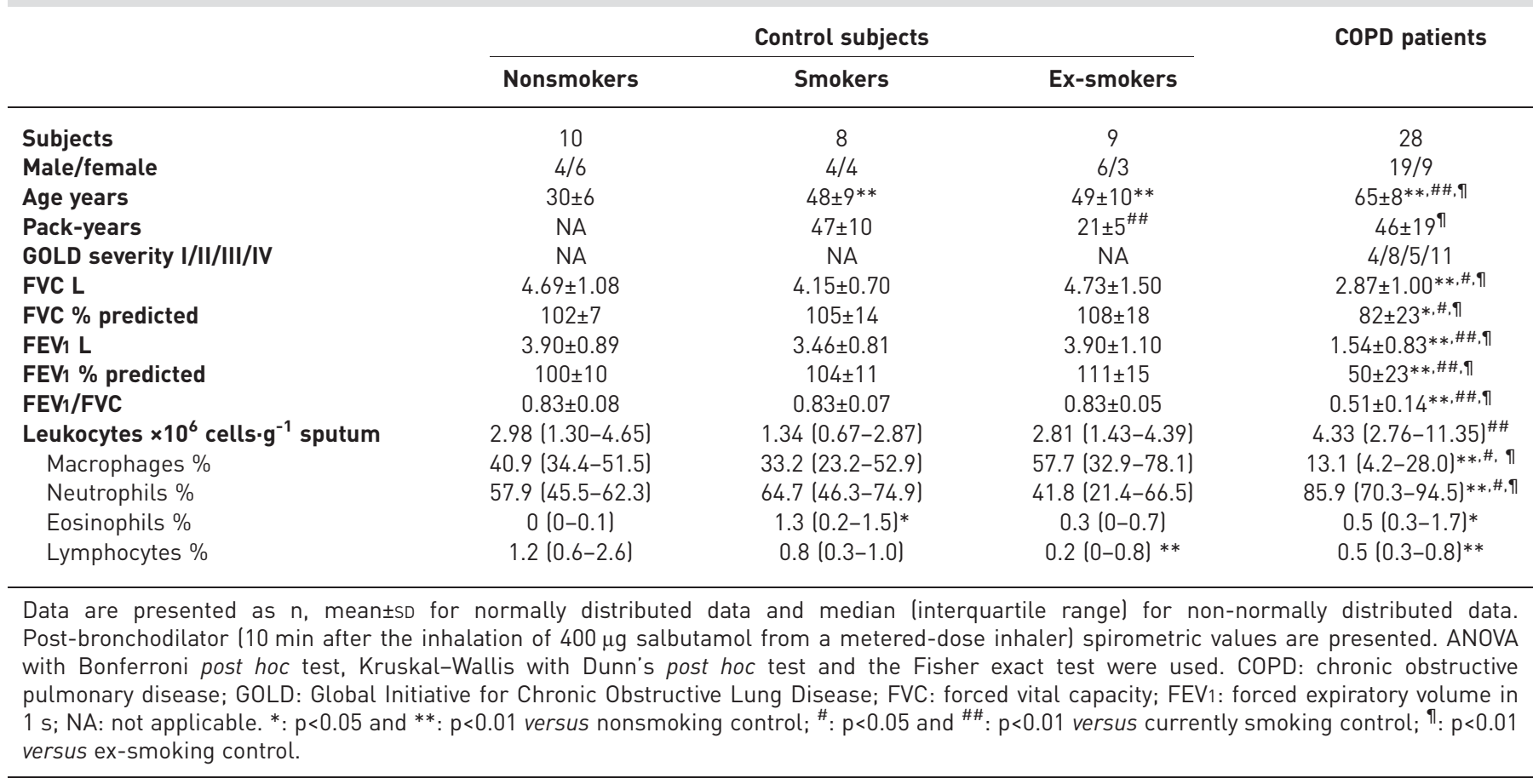

supernatant of wild-type mice exposed to the smoke of five cigarettes for three consecutive days compared to air-exposed controls (fig. 2a, b).

Besides inflammatory cells, pulmonary ATP concentration is also controlled by CD39 strongly expressed on airway epithelial cells [14]. Therefore, we further assessed the expression and pulmonary activity of membrane-bound ATPase in lung tissue of mice exposed to cigarette smoke for up to 28 days. Indeed, cigarette smoke exposure led to the strong upregulation of Cd39 mRNA (fig. 2c) as well as pulmonary ATPase activity in the lung tissue compared to air-exposed mice (fig. 2d-i). Interestingly, the highest ATPase activity was observed after 14 days of cigarette smoke exposure; however, pulmonary ATPase activity remained elevated on days 21 and 28. No ATPase activity was observed in lungs of CD39-deficient animals (online supplementary fig. S1).

Inhibition of the ectonucleotidase CD39 aggravates cigarette smoke-induced acute lung inflammation To evaluate the functional relevance of increased $C d 39$ expression due to smoke-induced lung inflammation, mice were treated intratracheally with the ectonucleotidase inhibitor ARL-67156 (100 mM) or vehicle $30 \mathrm{~min}$ before each smoke exposure. Compared to vehicle treatment ATP concentration was elevated in BALF of animals treated with ARL-67156, which was accompanied by an increase in macrophage and neutrophil counts and an elevation in the concentrations of interleukin (IL)-6, keratinocyte-derived chemokine (KC) and macrophage inflammatory protein (MIP)-2 in the BALF (fig. 3a-c).

\section{CD39 deficiency aggravates acute and chronic cigarette smoke-induced lung inflammation}

As a further proof of concept, we also exposed $C d 39^{-1-}$ mice to cigarette smoke for 3 days (five cigarettes per day). Again, CD39 deficiency was associated with an increase in BALF ATP levels, numbers of neutrophils and alveolar macrophages, as well as higher concentrations of IL-6, KC and MIP-2 compared to wild-type mice (fig. $4 \mathrm{a}-\mathrm{c}$ ).

In order to study the natural history and the pathological consequences leading to COPD, $C d 39^{-1-}$ mice were additionally examined in a model of chronic cigarette smoke induced-lung inflammation and lung emphysema. Following 4 months of cigarette smoke exposure in wild-type and $C d 39^{-/-}$mice, the average interalveolar distance (mean linear intercept, Lm) increased while the internal surface area of the lungs (ISA) decreased compared to air-exposed animals. These effects were significantly enhanced in smoke-exposed $\mathrm{Cd} 39^{-1-}$ mice compared to wild-type animals (fig. $4 \mathrm{~d}, \mathrm{e}$ ). 

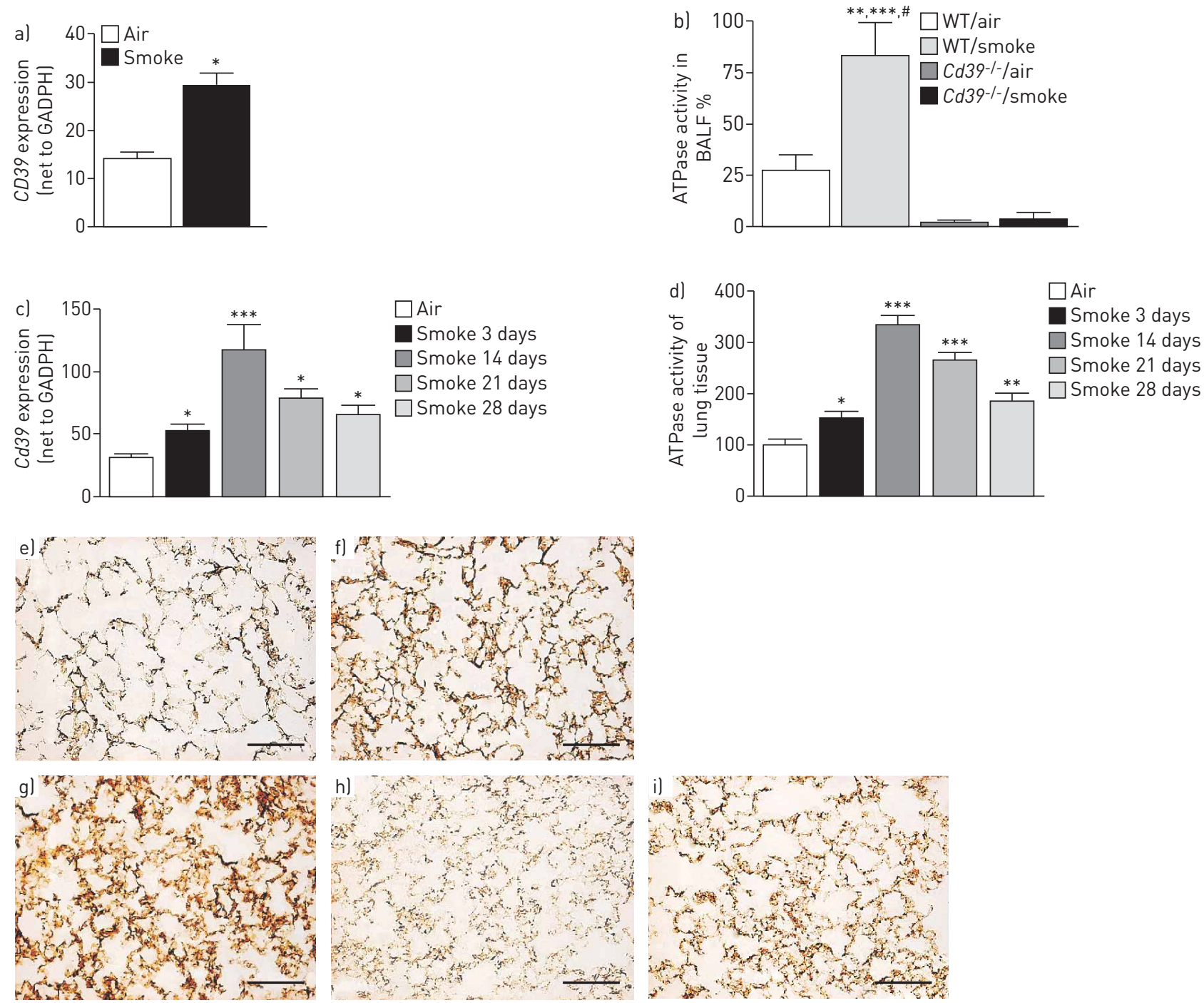

FIGURE 2 Pulmonary CD39 expression and ATPase activity are upregulated in a mouse model of acute cigarette smoke-induced lung inflammation. Male C57BL/6 mice were exposed to the smoke of five cigarettes or air on three consecutive days. $1 \mathrm{~h}$ after the last smoke exposure, bronchoalveolar lavage fluid (BALF) and lung tissue were collected to determine Cd39 mRNA expression and to assess ATPase activity. a) mRNA levels of Cd39 in BALF leukocytes of smoke- and air-exposed animals. Data are presented as mean \pm SEM; $n=6-8$ mice in each group; *: $p<0.05$. b) Freshly prepared cell-free BALF supernatant was incubated with $100 \mathrm{nM}$ ATP for $30 \mathrm{~min}$ at room temperature, and then ATP recovery was measured luminometrically. Data are presented as mean \pm SEM; $n=6-8$ mice in each group. ${ }^{* *}: \mathrm{p}<0.01$ wild-type (WT)/air versus WT/smoke; ${ }^{* * *}$ : $\mathrm{p}<0.001$ Cd39-/-/air versus WT/smoke; ${ }^{\#}$ : $p<0.01 \mathrm{Cd} 39^{-1-}$ /smoke versus WT/smoke. c) mRNA levels of $C d 39$ in total lung tissue of smoke- and air-exposed animals. Data are presented as mean \pm SEM; $n=6-8$ mice in each group; ${ }^{*}: p<0.05$ and ${ }^{* * *}: p<0.001$ mice exposed to smoke versus air. $d$-i) Pulmonary ATPase activity was assessed in WT mice exposed to air or cigarette smoke for 3-28 days. Lung sections were incubated with $300 \mathrm{mM}$ ATP for 90 min. d) Lead staining intensity of lung sections was quantified using ImageJ software (National Institutes of Health, Bethesda, MD, USA). Data are presented as mean \pm SEM; $\mathrm{n}=6-8$ mice in each group; ${ }^{*}: \mathrm{p}<0.05,{ }^{* *}: \mathrm{p}<0.01$ and ${ }^{* * *}: \mathrm{p}<0.001$ mice exposed to smoke versus air. e-i) Brown lead precipitate corresponds to ATPase activity in the lung tissue: e) exposure to air did not induce any ATPase activity in the lungs; f) enzyme activity was substantially increased upon smoke exposure already after 3 days; g) the highest level was observed at day 14 , gradually decreasing at h) day 21 and i) day 28 . Scale bars $=100 \mu \mathrm{m}$.

Therapeutic implication of a soluble ATPase in chronic cigarette smoke-induced emphysema and pulmonary inflammation

Our data suggest that endogenous CD39 activity is boosted and seems to partially protect mice and humans against further aggravation of cigarette smoke-induced lung inflammation. Therefore, we next questioned whether enhancing pulmonary ATPase activity might inhibit the development of cigarette smoke-induced lung emphysema. To this end, wild-type mice were exposed to cigarette smoke for 4 months (three cigarettes per day, 5 days a week). Afterwards mice received cigarette smoke for an additional 3 months with concomitant apyrase (grade VI, $200 \mathrm{~mL} 4 \mathrm{IU} \cdot \mathrm{mL}^{-1}$ intraperitoneally) or vehicle (once daily, 5 days per week). Similarly to the function of CD39, apyrase hydrolyses nucleoside triphosphates and diphosphates including ATP and ADP. Lungs were isolated to analyse mean linear intercept and the internal surface area 

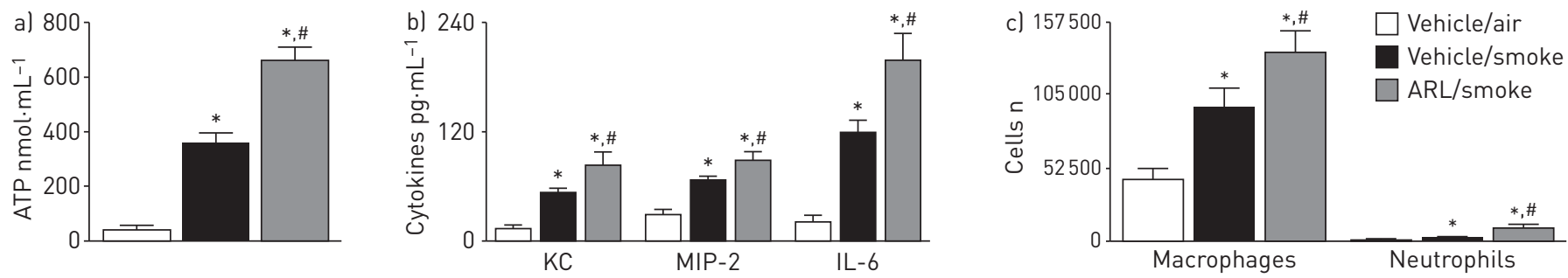

FIGURE 3 Inhibition of CD39 aggravates acute cigarette smoke-induced lung inflammation. Male C57BL/6 mice were exposed to air or the smoke of five cigarettes on three consecutive days. 30 min before smoke exposure, animals received an intratracheal injection of either vehicle or the CD39-inhibitor ARL 67156. $1 \mathrm{~h}$ after the last smoke exposure animals were killed and a) ATP concentrations; b) cytokine levels; and c) total with differential cell counts were analysed in bronchoalveolar lavage fluid. Data are presented as mean \pm SEM; $n=5$ mice in each group. KC: keratinocyte-derived chemokine; MIP: macrophage inflammatory protein; IL: interleukin. *: $p<0.05$ vehicle/smoke or ARL/smoke versus vehicle/air; \#: $\mathrm{p}<0.05 \mathrm{ARL} / \mathrm{smoke}$ versus vehicle/smoke.

of the lungs. As shown in table 3, administration of apyrase significantly decreased cigarette smoke-induced lung emphysema. Apyrase treatment also suppressed pulmonary inflammation as shown by the decreased BALF neutrophil and macrophage cell counts (fig. 5).

\section{Discussion}

Compelling evidence implicates an important role of the pulmonary ATP-P2 receptor axis in the pathogenesis of cigarette smoke-induced lung inflammation and emphysema in mice and men [3, 5, 7]. Extracellular pulmonary ATP levels are tightly regulated by the ectonucleotidase CD39/NTPDase1. Interestingly, CD39 deficiency has been linked to both decreased (e.g. allergic airway inflammation) and increased airway inflammation (such as in LPS-induced acute lung injury) $[14,20]$. Here we demonstrate that expressions of cellular CD39 (on BALF and sputum cells) as well as the activity of the soluble ectonucleotidase are elevated in the airways of patients with COPD. Increased CD39 activity/expression is maintained in patients with COPD even after smoking cessation. By using a translation mouse model, we can show that CD39 deficiency/inhibition is associated with the aggravation of cigarette smoke-induced lung inflammation and emphysema, while treatment of animals with exogenous CD39 rescues cigarette smoke-induced lung emphysema. Thus, our findings suggest that CD39 in the lungs has been upregulated, probably in a direct compensatory manner to dampen neutrophilic airway inflammation in COPD.

We previously demonstrated that cigarette smoke-induced lung inflammation in mice and humans is associated with an increase in pulmonary ATP levels, which correlates with disease severity and airway neutrophilia in COPD [3, 6]. It has also been shown that CD39 activity is decreased in tissue
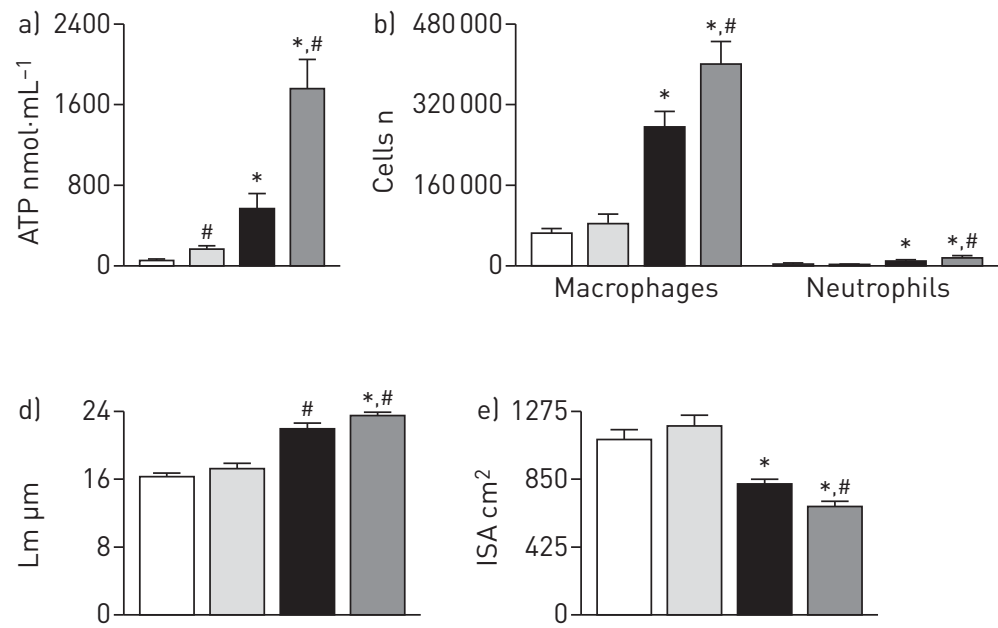

FIGURE $4 \mathrm{Cd} 39^{-/-}$mice display a more severe phenotype of acute and chronic cigarette smoke-induced lung inflammation and emphysema. a-c) Male $\mathrm{Cd} 39^{-1-}$ mice and wild-type (WT) animals were either exposed to room air or smoke of five cigarettes on each of three consecutive days. $1 \mathrm{~h}$ after the last smoke exposure animals were killed and bronchoalveolar lavage fluid was analysed for a) ATP concentration; b) the number and distribution of cells; and c) cytokine content. Data are presented as mean \pm SEM; $n=5$ mice in each group. $d$, el Age-matched male Cd39-/- or WT mice were exposed to air or smoke of three cigarettes 5 days a week for four consecutive months. Animals were sacrificed and the d) mean linear intercept (Lm) and e) internal surface area of the lungs (ISA) were measured ( $\mathrm{n}=8$ per group). KC: keratinocyte-derived chemokine; MIP: macrophage inflammatory protein; IL: interleukin. *: p<0.05 air versus cigarette smoke exposure; ${ }^{*}$ : $p<0.05$ smoke exposure of Cd39-/- versus WT animals. 
TABLE 3 Effect of a soluble ectonucleotidase on the progression of cigarette smoke-induced emphysema

\begin{tabular}{lcc} 
& $\begin{array}{c}\text { Mean linear } \\
\text { intercept } \mathbf{~ m m}\end{array}$ & $\begin{array}{c}\text { Internal surface } \\
\text { area of the lungs } \mathbf{c m}^{\mathbf{2}}\end{array}$ \\
\hline Air 4 months & $37.6 \pm 1.1$ & $1223 \pm 85$ \\
Smoke 4 months & $40.2 \pm 1.4^{*}$ & $1063 \pm 64^{*}$ \\
Air 7 months & $38.9 \pm 1.9$ & $1194 \pm 92$ \\
Smoke 7 months + vehicle & $43.4 \pm 1.4^{\#}$ & $995 \pm 18^{\#}$ \\
Smoke 7 months + apyrase & $40.0 \pm 1.3^{+}$ & $1161 \pm 61^{+}$
\end{tabular}

Data are presented as mean \pm SEM. Air: exposure to room air; smoke: exposure to cigarette smoke; apyrase/ vehicle: treatment with apyrase $\left(200 \mathrm{~mL} 4 \mathrm{IU} \cdot \mathrm{mL}^{-1}\right.$ intraperitoneally) or vehicle in the last 3 months of cigarette smoke exposure. *: $p<0.05$ air 4 months versus smoke 4 months; ${ }^{*}$ : $p<0.05$ air 7 months versus smoke 7 months + vehicle; ${ }^{+}: p<0.05$ smoke 7 months + apyrase versus smoke 7 months + vehicle.

compartments due to oxidative stress or pro-inflammatory cytokines such as tumour necrosis factor- $\alpha$ $[12,13,21]$. Indeed, allergic airway inflammation is associated with a decrease in pulmonary CD39 expression [14]. Surprisingly, we found an increase in CD39 expression of inflammatory cells and soluble ATPase activity in the airways of smokers and patients with COPD. Similar observations were also reported in platelets after acute smoke exposure $[22,23]$. However, in platelets an opposing effect of acute cigarette smoke stimulation on ATP hydrolysing capacity has also been noted [24]. In our translational mouse model, cigarette smoke exposure led to upregulation of CD39 in the lung tissue. Interestingly, a similar upregulation was previously reported in a mouse model of lipopolysaccharide (LPS)-induced acute lung injury (ALI) [20]. However, contradicting our observations, KRATZER et al. [25] reported that CD39 expression was significantly downregulated in lung tissue in rats exposed to second-hand smoke, and in lung tissue from COPD patients. The difference in animal models (direct versus second-hand smoke) and the compartmentalisation of the induction in CD39 activity in COPD patients (inflammatory cells versus lung tissue) might explain these seemingly contradictory findings.

Neutrophils play an important role both in ALI and cigarette smoke-induced lung inflammation, and binding of ATP to the $\mathrm{P}_{2} \mathrm{Y}_{2}$ receptor is responsible for neutrophil activation and recruitment to the side of inflammation [26]. Of note, it has been shown that CD39 regulates neutrophil recruitment [11] and LPS-induced IL-8 production [5, 12]. Interestingly, we also observed a correlation between CD39 activity and airway neutrophilia in COPD. Besides its essential role in activation of neutrophils, the ATP-P2 receptor axis also contributes to other fundamental pro-inflammatory cell functions, such as cell migration and/or mediator production (e.g. matrix metalloproteinase-9, reactive oxygen species, tissue inhibitor of metalloproteinase-1, IL-1 $\beta$ and IL-6) by macrophages, dendritic cells and airway epithelial cells $[3,6,27]$, known to be involved in the pathogenesis of COPD. The termination of ATP signalling by CD39 might be followed by the generation of extracellular adenosine, and consequently P1 receptor signalling, as studied by exhaled breath condensate measurements in COPD [28], which in many cases dampens acute inflammation and lung injury [5]. In summary, considering the crucial role of the ATP-P2 receptor

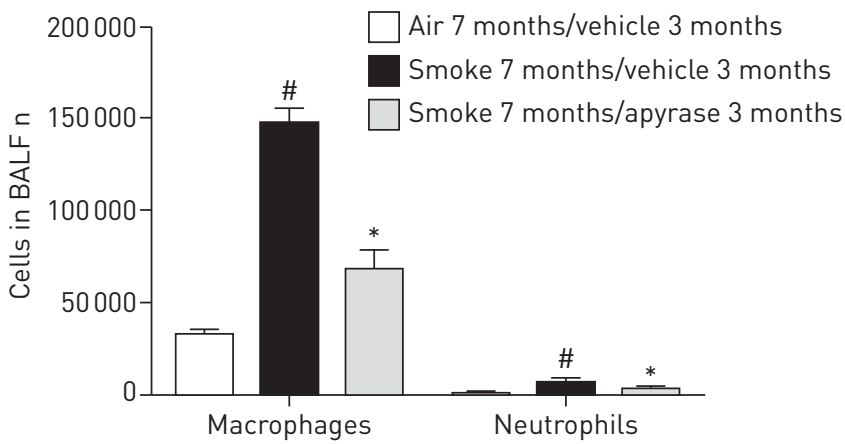

FIGURE 5 Effect of a soluble ectonucleotidase on cigarette smoke-induced chronic pulmonary inflammation. Male wild-type animals were either exposed to room air or cigarette smoke for 7 months with apyrase treatment (200 $\mathrm{mL} 4 \mathrm{IU} \cdot \mathrm{mL}^{-1}$ intraperitoneally) or vehicle in the last 3 months of cigarette smoke exposure. Bronchoalveolar lavage fluid (BALF) was analysed for the number of macrophages and neutrophils. Data are presented as mean ISEM. $\mathrm{n}=6$ per group. *: $\mathrm{p}<0.05$ smoke 7 months + apyrase versus smoke 7 months + vehicle; ${ }^{\#}: \mathrm{p}<0.05$ air 7 months versus smoke 7 months + vehicle. 
pathway in cigarette smoke lung inflammation, our results allow speculation that CD39 activity might be upregulated as a consequence of smoke-induced inflammation to modulate ATP-induced lung damage.

This hypothesis is supported by our finding that pulmonary application of the CD39 inhibitor ARL-67156 in wild-type mice significantly increases pulmonary ATP levels and aggravates acute cigarette smoke-induced lung inflammation, as determined by the increase in neutrophil and macrophage counts as well as by elevated levels of pro-inflammatory cytokines (IL-6, KC and MIP-2) in BALF. Accordingly, Cd39-/- mice display enhanced acute cigarette smoke-induced lung inflammation. These observations are in line with previous reports demonstrating that CD39 deficiency aggravates pulmonary neutrophilia and lung damage in ALI [20], while ARL-67156 treatment also intensifies allergic bronchospasm in guinea-pigs [29]. In addition, CD39 has been shown to regulate the ATP-P2X7 receptor-mediated leukocyte migration/recruitment and IL-1 $\beta$ production by macrophages [30,31]. Finally the ATP-CD39 axis is also involved in regulating cigarette smoke extract-induced production of IL-8/-6 by airway epithelia in vitro (authors' unpublished observation).

It is noteworthy that other soluble enzymes apart from CD39, namely pyrophosphatase/ phosphodiesterase- 1 and adenylate kinase- 1 are also involved in the metabolism of circulating ATP and ADP in serum $[32,33]$. However, we did not observe any substantive reconstitution of pulmonary ATP metabolism in cell-free BALF supernatants from CD39-deficient animals exposed to cigarette smoke or air (fig. 2b), but the function of membrane-bound enzymes in the lungs should still be explored. Nevertheless, our data suggest a major role for soluble CD39/NTPDase1 in the regulation of pulmonary ATP levels in cigarette smoke-induced lung inflammation.

Thus we provide evidence that smoke exposure leads to compensatory upregulation of cellular and soluble CD39 to alleviate cigarette smoke-induced lung inflammation. This observation is further supported by our histological findings that CD39 deficiency is accompanied by more severe emphysema in the model of chronic smoke exposure. In a similar manner to airway inflammation, a more severe form of colitis and aggravated hypoxic/ischaemic injury were reported in the CD39-deficient mouse [34, 35].

Finally, we demonstrate that apyrase, a functional analogue of CD39, limits the development of lung emphysema in mice with established cigarette smoke-associated lung inflammation. A similar beneficial effect of exogenous ATPase treatment has been reported for other inflammatory conditions, such as allergic airway inflammation and bronchospasm [18, 29], graft versus host disease [36] or colitis [37]. Despite all the limitations of such animal models, these data further strengthen our assumption that CD39 plays a protective role in cigarette smoke-associated lung inflammation and in COPD.

Our study also has limitations. NTPDase1/CD39 is expressed in human epithelial cells, playing a role in the regulation of leukocyte infiltration [38]. In the human study we measured CD39 expression and ATPase activity in sputum and BALF inflammatory cells, but not in airway epithelium. However, in the murine model we clearly show that ATPase activity in lung tissue is heightened after smoke exposure, which suggests that airway epithelium is involved in the regulation of airway ATP in smoking control subjects. This needs further clarification by ex vivo studies on human bronchial epithelial cell cultures. We also acknowledge that the COPD patients were older than the control subjects. Nonetheless, no correlation between age and CD39 expression was observed in normal circulating leukocytes [39]. Lastly, apyrase, a soluble ATPase, was administered intraperitoneally in the murine model of chronic smoke exposure; therefore, besides pulmonary function, its protective role on the development of emphysema may also reflect apyrase activities in the systemic circulation.

In summary, we demonstrate that CD39 functionality is enhanced in cigarette smoke-induced airway inflammation in humans and mice and may play a compensatory role in this setting, as null mice suffer more severe injury. More importantly, treatment with soluble CD39 ameliorates the development of cigarette smoke-induced emphysema. Our findings further underline the significance of P2 receptor signalling in the pathomechanism of COPD, which can be modulated beneficially by therapeutic agents targeting ATP hydrolysis.

\section{Acknowledgements}

The authors would like to thank Jonas Schupp, Wolfram Meschede, Marcio Jose Dias Amorim and Dorota Rombach (Dept of Pulmonary Medicine, University Hospital Freiburg, Freiburg, Germany) for their assistance with patient recruitment.

\section{References}

1 Rabe KF, Hurd S, Anzueto A, et al. Global strategy for the diagnosis, management, and prevention of chronic obstructive pulmonary disease: GOLD executive summary. Am J Respir Crit Care Med 2007; 176: 532-555.

2 Lázár Z, Vass G, Huszár E, et al. Exhaled breath condensate: adenosine, ATP and other purines. In: Horvath I and de Jongste JC, eds. Exhaled Biomarkers (ERS Monograph). Sheffield, European Respiratory Society, 2010; pp. 183-195.

3 Lommatzsch M, Cicko S, Müller T, et al. Extracellular adenosine triphosphate and chronic obstructive pulmonary disease. Am J Respir Crit Care Med 2010; 181: 928-934. 
4 Esther CR Jr, Alexis NE, Clas ML, et al. Extracellular purines are biomarkers of neutrophilic airway inflammation. Eur Respir J 2008; 31: 949-956.

5 Idzko M, Ferrari D, Eltzschig HK. Nucleotide signalling during inflammation. Nature 2014; 509: 310-317.

6 Cicko S, Lucattelli M, Müller T, et al. Purinergic receptor inhibition prevents the development of smoke-induced lung injury and emphysema. J Immunol 2010; 185: 688-697.

7 Lucattelli M, Cicko S, Müller T, et al. P2X7 receptor signaling in the pathogenesis of smoke-induced lung inflammation and emphysema. Am J Respir Cell Mol Biol 2011; 44: 423-429.

8 Robson SC, Sévigny J, Zimmermann H. The E-NTPDase family of ectonucleotidases: structure function relationships and pathophysiological significance. Purinergic Signal 2006; 2: 409-430.

9 Picher M. Mechanisms regulating airway nucleotides. Subcell Biochem 2011; 55: 17-49.

10 Mizumoto N, Kumamoto T, Robson SC, et al. CD39 is the dominant Langerhans cell-associated ecto-NTPDase: modulatory roles in inflammation and immune responsiveness. Nat Med 2002; 8: 358-365.

11 Corriden R, Chen Y, Inoue Y, et al. Ecto-nucleoside triphosphate diphosphohydrolase 1 (E-NTPDase1/CD39) regulates neutrophil chemotaxis by hydrolyzing released ATP to adenosine. J Biol Chem 2008; 283: 28480-28486.

12 Eltzschig HK, Sitkovsky MV, Robson SC. Purinergic signaling during inflammation. N Engl J Med 2012; 367: 2322-2333.

13 Antonioli L, Pacher P, Vizi ES, et al. CD39 and CD73 in immunity and inflammation. Trends Mol Med 2013; 19: 355-367.

14 Idzko M, K Ayata C, Müller T, et al. Attenuated allergic airway inflammation in Cd39 null mice. Allergy 2013; 68: $472-480$.

15 Djukanović R, Sterk PJ, Fahy JV, et al. Standardised methodology of sputum induction and processing. Eur Respir J 2002; 37: Suppl. 1s-2s.

16 Cavarra E, Lucattelli M, Gambelli F, et al. Human SLPI inactivation after cigarette smoke exposure in a new in vivo model of pulmonary oxidative stress. Am J Physiol Lung Cell Mol Physiol 2001; 281: L412-L417.

17 Langer D, Hammer K, Koszalka P, et al. Distribution of ectonucleotidases in the rodent brain revisited. Cell Tissue Res 2008; 334: 199-217.

18 Idzko M, Hammad $\mathrm{H}$, van Nimwegen $\mathrm{M}$, et al. Extracellular ATP triggers and maintains asthmatic airway inflammation by activating dendritic cells. Nat Med 2007; 13: 913-919.

19 Ayata CK, Ganal SC, Hockenjos B, et al. Purinergic $\mathrm{P}_{2} \mathrm{Y}_{2}$ receptors promote neutrophil infiltration and hepatocyte death in mice with acute liver injury. Gastroenterology 2012; 143: 1620-1629.

20 Reutershan J, Vollmer I, Stark S, et al. Adenosine and inflammation: CD39 and CD73 are critical mediators in LPS-induced PMN trafficking into the lungs. FASEB J 2009; 23: 473-482.

21 Vlaar AP, van Son WJ, Bakker WW. Histochemical detection of ischemia-like alterations induced in kidney tissue in vitro - different sensitivity to oxidant stress of glomerular ENTPD1 versus E5NT. Nephron Physiol 2009; 111: $1-8$.

22 Thomé GR, Mazzanti CM, Ahmed M, et al. Activity of ectonucleotidases and adenosine deaminase in rats exposed to cigarette smoke. Inhal Toxicol 2009; 21: 906-912.

23 dos Santos Jaques JA, Ruchel JB, Schlemmer KB, et al. Effects of curcumin on the activities of the enzymes that hydrolyse adenine nucleotides in platelets from cigarette smoke-exposed rats. Cell Biochem Funct 2011; 29: $630-635$.

24 Togna AR, Latina V, Orlando R, et al. Cigarette smoke inhibits adenine nucleotide hydrolysis by human platelets. Platelets 2008; 19: 537-542.

25 Kratzer A, Salys J, Sévigny J, et al. Second hand smoke exposure impairs CD39 expression and function in the lung. Eur Respir J 2012; 40: Suppl. 56, P1429.

26 Chen Y, Corriden R, Inoue Y, et al. ATP release guides neutrophil chemotaxis via P2Y2 and A3 receptors. Science 2006; 314: 1792-1795.

27 Myrtek D, Müller T, Geyer V, et al. Activation of human alveolar macrophages via P2 receptors: coupling to intracellular $\mathrm{Ca}^{2+}$ increases and cytokine secretion. J Immunol 2008; 181: 2181-2188.

28 Esther CR Jr, Lazaar AL, Bordonali E, et al. Elevated airway purines in COPD. Chest 2011; 140: 954-960.

29 Chávez J, Vargas MH, Rebollar-Ayala DC, et al. Inhibition of extracellular nucleotides hydrolysis intensifies the allergic bronchospasm. A novel protective role of ectonucleotidases. Allergy 2013; 68: 462-471.

30 Hyman MC, Petrovic-Djergovic D, Visovatti SH, et al. Self-regulation of inflammatory cell trafficking in mice by the leukocyte surface apyrase CD39. J Clin Invest 2009; 119: 1136-1149.

31 Lévesque SA, Kukulski F, Enjyoji K, et al. NTPDase1 governs P2X7-dependent functions in murine macrophages. Eur J Immunol 2010; 40: 1473-1485.

32 Yegutkin GG, Samburski SS, Mortensen SP, et al. Intravascular ADP and soluble nucleotidases contribute to acute prothrombotic state during vigorous exercise in humans. J Physiol 2007; 579: 553-564.

33 Yegutkin GG, Wieringa B, Robson SC, et al. Metabolism of circulating ADP in the bloodstream is mediated via integrated actions of soluble adenylate kinase-1 and NTPDase1/CD39 activities. FASEB J 2012; 26: 3875-3883.

34 Friedman DJ, Künzli BM, A-Rahim YI, et al. CD39 deletion exacerbates experimental murine colitis and human polymorphisms increase susceptibility to inflammatory bowel disease. Proc Natl Acad Sci USA 2009; 106: $16788-16793$.

35 Eltzschig HK, Köhler D, Eckle T, et al. Central role of Sp1-regulated CD39 in hypoxia/ischemia protection. Blood 2009; 113: 224-232.

36 Wilhelm K, Ganesan J, Müller T, et al. Graft-versus-host disease is enhanced by extracellular ATP activating P2X7R. Nat Med 2010; 16: 1434-1438.

37 Atarashi K, Nishimura J, Shima T, et al. ATP drives lamina propria T(H)17 cell differentiation. Nature 2008; 455: 808-812.

38 Fausther M, Pelletier J, Ribeiro CM, et al. Cystic fibrosis remodels the regulation of purinergic signaling by NTPDase1 (CD39) and NTPDase3. Am J Physiol Lung Cell Mol Physiol 2010; 298: L804-L818.

39 Pulte ED, Broekman MJ, Olson KE, et al. CD39/NTPDase-1 activity and expression in normal leukocytes. Thromb Res 2007; 121: 309-317. 